55 UNEXPECTED DEVELOPMENT OF HEARING LOSS IN HIGH RISK D5 NEONATES. Toni A. Nield, Shirley Schrier, Angela Hospital of Los Angeles, Div. Neonatology and Pediatric Pulmonology, Div. of Hearing and Speech, Los Angeles.

Seven high risk neonates who passed initial brain stem auditory evoked response (BAER) testing had hearing loss on subsequent audiologic evaluation. Gestational ages were $36 \pm 5$ weeks $(M \pm S D)$ with birthweights $2483 \pm 975$ gms. Clinical problems which they had in common included: lung disease requiring ventilation for 1 month; persistent fetal circulation; evidence of CNS insult (5 abnormal EEG's, 4 seizures, 2 hydrocephalus, and 1 documented parenchymal bleed). Al1 received the following medications: ampicillin, gentamicin, furosemide, chlorothiazide, isoproterenol, and pancuronium.

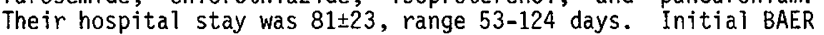
testing using "click" stimuli at $60 \mathrm{~dB}$ and $30 \mathrm{~dB}$ in each ear was done at the time of discharge. (All infants were past term by done at the time of discharge. (All infants were past term by
corrected age.) Wave $V$ responses occurred at latencies within the range of 7.5 to $8.5 \mathrm{msec}$ at $60 \mathrm{~dB}$ and 8.5 to $9.5 \mathrm{msec}$ at $30 \mathrm{~dB}$ (within normal limits). Audiologic evaluation using Conditioned Orientation Response (COR) was done at a mean age of 18 \pm 3 , range 13-20 months. The level of hearing loss in al1 7 children was severe to profound $(>80 \mathrm{~dB})$. We conclude that infants who have been very $i 11$ during the neonatal period, including term infants, may remain at risk for development of subsequent hearing loss even though they have passed a BAER test at $1-3$ months of age.

ANXIETY IN MOTHERS OF MONITORED INFANTS (MOMI) AND

56 FATHERS OF MONITORED INFANTS (FOMI). Dilip Purohit, Conway Saylor, Margaret Ford, Susan Crocker (Spon. by Paul Gillette), Medical University of South Carolina, De

of Pediatrics and Psychlaty whose infants were monitored at home for apnea. An adapted version of the "State" portion of the State-Trait Anxiety Inventory (STAI) was employed to assess parent's anxiety pertaining to their infants sleeping alone in another room and sleeping in the same room with the parents. The Trait portion of the STAI served as a measure of general anxiety. Twenty-five MOMI, as well as 9 motiers of full-term or premature nonmonitored infants (NonMOMI), were asked to complete the questionnaire at discharge and at 1 and 3 months pos.t discharge. Six FOMI also completed the questionnaire at discharge.

The preliminary results indicate the following: 1) At discharge, MOMI reported significantly higher anxiety than the NonMOMI, around the issue of infants sleeping alone $(t=2.73, p<.01)$, and higher anxiety around the issue of infants sleeping under their direct supervision. 2) For both situations, differences in anxiety between groups decreased at 1 month and nearly disappeared at 3 months. 3) Groups did not differ on "Trait" or general anxiety at any of the 3 intervals, and the general level of anxiety: remained stable across trials. 4) Father's anxiety level for the situations namely, the child with him and the child sleeping alone, correlated with the mothers anxiety in all situations ( $r=$ $.78-.99)$ and mothers anxiety of the same situation $(r=.99)$, re-
respectively. However, the father's general anxiety level did not respectively. However, the father's general anxiety
correlate with any measures of the mother's anxiety.

SEXUAL, VIOLENCE AND PLACEMENT OF CHILDREN, Dianne O. Regan 57 Seunde $\mathrm{K}$. Fhr1ich and Loretta P. Finnegan, Jefferson Medica Soundra M. Ehrlich and Loretta P. Finnegan, Jefferson Medical trics, Philadelphia, PA.

Family Center is a comprehensive program which provides obstetrical and psychosocial services and methadone maintenance for pregnant drug-dependent women and their infants. Between 1979 and 1983, 171 women on enrollment to the program completed our Violence Questionnaire. Objectives of the questionnaire were: 1) to ascertain episodes and degrees of violence experienced by the women, including acts of physical and sexual abuse occurring in childhood or as an adult. 2) to learn if women reporting a history of violence/abuse were more likely to have had children in foster care. Individual item responses on the questionnaire, reported in percentages, were:

QUESTION ITEMS \begin{tabular}{|l|l|l|l|l|l} 
& Raped As & Molested & Raped & Beaten As & Beaten As
\end{tabular}

$\%$ of 171

$\%$ Child | Adut

as Child $>$ once

Foster Care (40)

(31)

With Mother $(60)$

$$
\mathrm{p}=<.01
$$$$
46
$$

$46 \quad 23$

\begin{tabular}{l|l}
$77^{*}$ & \\
23 & 81,9 \\
\hline
\end{tabular}

31

\begin{tabular}{|c|c} 
Adult & Child \& Adult \\
\hline$(65)$ & $(15)$ \\
41 & 38 \\
59 & 62
\end{tabular}

of the 171 women studied, $40 \%$ had childiren in voluntary or involuntary foster placement. Women with a reported history of sexual trama, particularly if occuring in childhood or repeatedly, were significantly more likely to have children in foster care $(\mathrm{p}=<.01)$. Women who were physically abused (without sexual trama) as children and/or adults were less likely to have their children in placement. This study suggests that failure to resolve childhood sexual trauma or coping with the trauma by use of illicit drugs, dismupts the ability of women to parent their own children. The effects of violence toward women, particularly when they themselves were children, may have untoward effects upon their own children.
OUTCOME OF INFANTS WITH BIRTHWEIGHTS $<800$ GRAMS. Seetha 58 Shankaran, Mary P. Bedard, Eunice Woldt, Thomas Koep of Med. and Children's Hosp of Mich., Dept. of Ped., Detroit, MI A prospective study of 14 outborn infants $<800 \mathrm{~g}$ at birth and transferred $<24 \mathrm{hrs}$ of age to a tertiary neonatal intensive care unit was conducted to determine neurologic and developmental outcome. Mean birthweight was $750 \mathrm{~g} \pm 69 \mathrm{~g}$ and mean gestational age was $28+2$ wks. $8 / 14$ were small for gestational age and 6 were male. $5 / 14$ infants required ventilatory support for $>30$ days and $3 / 14$ had moderate or severe intracranial hemorrhage. Mean age at follow-up was $39 \pm 10$ mos (range 18-48). Growth measurements were $<5$ percentile in $3 / 14$ for weight, $1 / 14$ for height, and 4/14 for head circumference. There were no significant differences in growth between $A G A$ and SGA infants.

The mean Bayley mental score for 3 infants $<30$ months was 85 and the mean motor score was 87. The mean score on the General Cognitive Index on the Mccarthy scale for 8 infants was $84 \pm$ 14.7. Subtest means were: verbal $40+8$, perceptual $44+7.4$ quantitative $40+10.5$, memory $40+6.1$, and motor $44.5+6.1$. Two infants with visual deficits were not tested. Neurologic sequelae were present in 2 infants, one with generalized hypotonia and one with a left hemiparesis. Visual deficits occurred in 2 patients, impaired hearing in 1 , and impaired speech in 3 . Neurologic outcome was not related to the duration of mechanical ventilation, severity of intracranial hemorrhage or intrauterine growth.

This study indicates that outcomes of infants with birth weight $<800 \mathrm{~g}$ who are outborn is much improved.

THE YALE CHILDRENS INVENTORY (YCI) : A NEWLY

59 DEVELOPED INSTRUMENT TO ASSESS CHILDREN WITH ATTENTIONAL DEFICITS AND LEARNING PROBLEMS. Sally E. Shaywitz, Carla Schnell, and Bennett A. Shaywitz, Yale Med, Sch. Depts. Ped. \& Child Stdy Ctr. questionnaire with DSM III criteria as its core, is designed for pediatricians to diagnose disorders characterized by academic difficulties, hyperactivity or attentional dysfunction. Scale construction resulted in 11 reliable narrow band scales (coefficient alpha internal consistency, $\bar{x}=.85, .72-$ .93 ; test-retest $\bar{x}=.79, .61-.89)$. Second order factor analysis yielded two broad bands: Behavior and Cognitive with Attention the only narrow band scale to load highly on both broad bands, providing empiric support to the notion that attentional problems are central to both behavioral and cognitive difficulties, and supporting DSM III nosology for Attention Deficit Disorder (ADD). A discriminant function based on the YCI scales classified normal and learning disabled children with a sensitivity of $82 \%$ and a specificity children with a sensitivity of 828 and a specificity of $96 \%$. Differential relationships of the YCI scales
with a series of external criterion measures provides with a series of external criterion measures provides years later). Based on DSM III criteria and demonstrating reliability and validity, the YCI represents a significant advance in the diagnosis and learning problems.

\section{DEVELOPMENTAL OUTCOME IN INFANT TRACHEOSTOMY} orlowich \& Children's Hospital, Dept. of Pediatrics., Cleveland, Ohio. Twenty-one survivors of long-term tracheostomy in infancy were seen for developmental follow-up at a mean age of 4.7 years $(S D=1.6)$. Measures of intellectual ability (standardized IQ tests), growth (weight for age), and social and behavioral competence based on Ch1ld Behavior Checklist (CBC) were taken The sample was primarily white $(67 \%)$, male (57\%) and middle class with a mean length of tracheostomy of 44 months $(S D=31)$. Almost half $(45 \%)$ were prematures with $30 \%$ VLBW. One-quarter had some indication of neurological damage, although children with documentable mental retardation were excluded from follow-up. The group as a whole achieved a mean Full scale IQ of $89.9(\mathrm{SD}=16)$, in the low normal range. Mean percentile of weight for age was $42.6(S D=24)$. The majority of children showed significant behavioral and social problems with $70 \%$ scoring in the deviant range on at least one subscale of the CBC. Correlational analyses indicated that, within this sample, prematurity was associated with lowered IQ $(r=.59$, $p<.003)$ and poorer behavioral functioning $(r=.42, p<.05)$ Neurological impairment $(x=.49, p<.03)$ and socioeconomic status $(r=.68, p<.002)$ were associated with behavioral difficulties, but not intellectual problems. The present data suggest that the impact of infant tracheostomy on later development is complex and intera 